\title{
ESTRESSE EM PACIENTES COM DIABETES TIPO 2
}

\section{Stress in patients with type 2 diabetes}

Natália Colombo Ferreira ${ }^{1}$, Daniela Vicente Bavaresco ${ }^{1}$, Luciane Bisognin Ceretta ${ }^{1,2}$, Lisiane Tuon ${ }^{1,2}$, Karin Martins Gomes ${ }^{2}$, Graziela Amboni²

${ }^{1}$ Programa de Residência Multiprofissional em Atenção Básica/Saúde da Família Universidade do Extremo Sul Catarinense - UNESC, Criciúma, SC, Brasil.

2 Programa de Pós-Graduação em Saúde Coletiva (Mestrado Profissional) Universidade do Extremo Sul Catarinense - UNESC, Criciúma, SC, Brasil.

\section{Endereço para correspondência:}

Graziela Amboni

Programa de Residência Multiprofissional em Atenção Básica/Saúde da Família Universidade do Extremo Sul Catarinense - UNESC

Avenida Universitária, 1105

Criciúma - SC

CEP - 88806-000

Email: gam@unesc.net 


\title{
Resumo
}

Este artigo objetiva apresentar uma revisão sobre a relação entre o Estresse e Diabetes do tipo 2. Foi realizada uma revisão de literatura narrativa na base de dados da biblioteca Virtual em Saúde por artigos publicados entre os anos de $2003 \mathrm{e}$ 2013, utilizando os descritores "estresse e diabetes tipo 2" e suas combinações. Os achados desta revisão reforçam que há relação entre estresse e diabetes Os sintomas do estresse mais citados foram os psicológicos, sendo que a predominância do estresse foi maior nas mulheres que nos homens. Foi possível inferir que as mulheres com estresse elevado apresentaram controle glicêmico alterado. A maioria relatou sofrimento decorrente de problemas nas relações interpessoais, óbito, doença de familiares e solidão. Os resultados ainda sugerem que outros fatores, além dos emocionais, devem ser considerados na análise da adesão ao tratamento do diabetes. Houve maior proporção de trabalhadores diabéticos com sobrepeso, hipertensão arterial e glicemia capilar alterada, quando comparados aos que não tinham essa doença, ficando evidente que pacientes com doenças crônicas têm maior probabilidade de desenvolver formas patológicas de estresse, ansiedade e depressão, podendo apresentar ainda dificuldades no manejo da doença comprometendo a adesão ao tratamento e consequentemente sua qualidade de vida. Nossos resultados mostram a relação do estresse como fator associado à portadora de $\mathrm{DM}$ e a necessidade de medidas que incluam o controle de estresse ao tratamento preconizado para esta doença, com a finalidade de melhorar os níveis glicêmicos e prevenir o aparecimento de complicações.

Palavras-chave: Estresse; Diabetes mellitus; Psicologia; Saúde Coletiva.

\begin{abstract}
This paper presents a review of the relationship between stress and diabetes type 2 . Narrative review of the literature was performed in the database of the Virtual Health Library for articles published between 2003 and 2013, using the keywords "stress and diabetes type 2" and combinations thereof. This review's findings reinforce that there is a relationship between stress and diabetes, the symptoms were the most cited psychological stress, with stress and prevalence was higher in women than in men. There was possible to infer that women with high stress had altered glycemic
\end{abstract}




\section{Revisão}

\section{Saúde e Processos Psicossociais}

control. Most reported suffering due to problems in interpersonal relationships, death, family illness and loneliness. The results also suggest that other factors, besides the emotional, should be considered in the analysis of treatment compliance diabetes. It was higher proportion of workers with diabetes: overweight; hypertension and abnormal blood glucose compared to those without this disease is quite evident that patients with chronic diseases are more likely to develop pathological forms of stress, anxiety and depression, and may also present difficulties in management of the disease affecting adherence to treatment and consequently their quality of life. Our results show the relationship of stress factor associated with carriers of DM and the need to include control measures that stress the recommended treatment for this disease, with the aim of improving blood glucose levels and prevent the onset of complications.

Keywords: Stress; diabetes mellitus; psychology; Public Health.

\section{INTRODUÇÃO}

O Diabetes Mellitus (DM) é uma doença que está se tornando cada vez mais comum e com crescente incidência. Apresenta alta morbimortalidade, com perda importante na qualidade de vida e é uma das principais causas de mortalidade, insuficiência renal, amputação de membros inferiores, cegueira e doença cardiovascular ${ }^{1}$.

O Ministério da Saúde classifica o diabetes como sendo um grupo de doenças metabólicas caracterizadas por hiperglicemia e associadas a complicações, disfunções e insuficiência de vários órgãos, especialmente olhos, rins, nervos, cérebro, coração e vasos sanguíneos. Podendo resultar de falhas de secreção e/ou ação da insulina envolvendo processos de destruição das células beta do pâncreas (produtoras de insulina), resistência à ação da insulina, distúrbios da secreção da insulina, entre outros ${ }^{1}$.

Segundo Zagury et al. ${ }^{2}$, o diabetes pode ser ocasionado também por cirurgias, estresse, alimentação rica em carboidratos concentrados - como balas, doces, açúcar -, menopausa e certos medicamentos. Há hipóteses de que fatores psicológicos possam produzir sintomas de diabetes. $\mathrm{O}$ autor ainda relata que $\mathrm{O}$ 


\section{Revisão}

\section{Saúde e Processos Psicossociais}

estresse também é visto como um dos fatores que podem dificultar o controle dos níveis de glicose e até mesmo levar a um quadro de hiperglicemia, devido aos hormônios produzidos nessas situações. No entanto, o estresse é positivo e auxilia quando permite ao paciente enxergar a importância da adesão ao tratamento. Segundo Santos $^{3}$, o estresse pode causar a destruição imunológica das células beta do pâncreas, causando deficiência na produção de insulina, que deve ser administrada pelo paciente neste caso.

O conviver com o Diabetes Mellitus incorre em constantes adaptações e reestruturações das formas de viver que podem ser geradoras de estresse e sofrimento. O estresse envolve um conjunto de reações físicas corporais e emocionais em respostas a eventos vivenciados pelos seres humanos. O descobrimento de doenças gera a necessidade de adaptação de rotinas e modos de vida, sendo que esta percepção e a intensidade das reações variam para cada indivíduo 4 .

O Diabetes Mellitus configura-se hoje como uma epidemia mundial. Assim, o envelhecimento da população, a crescente urbanização e a mudança para estilos de vida pouco saudáveis são os principais responsáveis pelo aumento da incidência e prevalência do diabetes em todo o mundo ${ }^{1}$.

É uma doença crônica, caracterizada pela elevação da glicose (açúcar) no sangue acima da taxa normal (hiperglicemia). A taxa normal é de aproximadamente 60 a $110 \mathrm{mg} \%$. Ele é causado por fatores genéticos (herdados) e ambientais, isto é, a pessoa quando nasce já traz consigo a possibilidade de ficar diabética. Quando, aliado a isso, ocorrem fatores como obesidade, infecções bacterianas e viróticas, traumas emocionais, gravidez, entre outros, a doença pode surgir mais cedo².

Dados brasileiros mostram que as taxas de mortalidade por DM (por 1000 habitantes) apresentam relevante aumento com o progredir da idade, variando de 0,46 para a faixa etária de 0 a 29 anos de idade a 223,3 para a de 60 anos ou mais, ou seja, um gradiente 400 vezes maior se comparado as taxas ${ }^{1}$.

Estima-se que a prevalência de diabetes, em 2000, tenha sido de 2,8\%, afetando 171 milhões de pessoas em todo o mundo, e espera-se para 2030 a prevalência de 4,4\%, o que corresponderia a 377 milhões de indivíduos diabéticos ${ }^{5}$.

De acordo com Brasi ${ }^{1}$, mundialmente os custos diretos para o atendimento ao diabetes variam de $2,5 \%$ a $15 \%$ dos gastos nacionais em saúde, dependendo da 


\section{Revisão}

\section{Saúde e Processos Psicossociais}

prevalência local de diabetes e da complexidade do tratamento disponível. Além dos custos financeiros, o diabetes acarreta também outros custos associados à dor, ansiedade, inconveniência e menor qualidade de vida que afeta doentes e suas famílias. O diabetes representa também carga adicional à sociedade, em decorrência da perda de produtividade no trabalho, aposentadoria precoce e mortalidade prematura.

O número de diabéticos no Brasil segundo a Sociedade Brasileira de Diabetes ${ }^{6}$ é de aproximadamente 12.054.827 (doze milhões, cinquenta e quatro mil, oitocentas e vinte e sete pessoas), ou $6,2 \%$ da população. Tendo como estimativa do número de diabéticos adultos (20-79 anos), no Brasil, 11.933.580, com prevalência nacional da doença de 9,04\%. Em 2013 morreram 124.687 (20-79 anos) relacionados ao diabetes. Segundo o Ministério da Saúde ${ }^{1}, 500$ novos casos de diabetes são diagnosticados todos os dias no Brasil (através do serviço público de saúde).

O diabetes é uma doença ainda sem tratamento definitivamente satisfatório, não sendo possível evitar totalmente o desenvolvimento de complicações crônicas nestes doentes. Surge, portanto, uma inquietação envolvendo a área da psicologia, preocupada com a reparação do que é desagradável e negativo na vida dos pacientes diabéticos, buscando compreender, auxiliar e contribuir para que sintam que vale a pena envolverem-se ativamente na gestão da sua doença, colaborando na prevenção das complicações crônicas ${ }^{7}$.

Segundo Penteado ${ }^{8}$, não se tem ideia da prevalência do estresse no Brasil e as taxas de morbimortalidade a ele associadas. O perfil psicológico e o grau de aceitação do DM parecem influenciar diretamente os níveis glicêmicos. A avaliação do estresse vem se tornando cada vez mais importante para medir o impacto geral da DM e suas complicações, podendo dar ao paciente apoio adequado no tratamento e auxílio para uma melhor qualidade de vida.

Assim, este artigo objetiva apresentar uma revisão sobre a relação entre o Estresse e o Diabetes do tipo 2. 
METODOLOGIA

Foi realizada uma revisão de literatura narrativa na base de dados da biblioteca Virtual em Saúde (BVS), por artigos publicados entre os anos de 2003 e 2013, utilizando os descritores "estresse e diabetes tipo 2" e suas combinações. A seleção dos artigos considerou apenas artigos nacionais e aqueles mais relevantes de acordo com a abrangência do tema proposto. Foram encontrados aproximadamente 20 artigos nacionais, e desses, 6 artigos preencheram os critérios de inclusão.

\section{RESULTADOS}

Recentemente algumas pesquisas têm sido realizadas tendo como foco a relação entre diabetes e estresse. Um estudo recente desenvolvido por Cantos ${ }^{9}$ avaliou dois pacientes diabéticos, sobre alguns indicadores da resposta de estresse por meio dos questionários do estado de estresse ${ }^{10}$, tolerância ao estresse ${ }^{11}$, vulnerabilidade ao estresse ${ }^{12}$ e consciência do estresse ${ }^{13}$. Uma avaliação do estado físico foi realizada por um questionário proposto por Boucher ${ }^{14}$.

Os resultados mostraram que, em todas as ações, o diálogo e a interação favoreceram a promoção de saúde na busca de uma melhor qualidade de vida dos pacientes. Por meio da análise dos questionários sobre estresse, notaram-se melhoras no estado emocional dos pacientes, fato que talvez possa ser atribuído à mudança no estilo de vida, no hábito alimentar e no incentivo à prática de exercício físico, bem como ao abandono de hábitos prejudiciais, como o tabagismo, o sedentarismo, entre outros. No que concerne aos fatores mais valorizados, constataram-se a interação dos pacientes com o grupo, a interação destes com a equipe multiprofissional de saúde e o acolhimento da equipe.

Um outro estudo desenvolvido por Ramos ${ }^{15}$ verificou a relação entre fatores emocionais e adesão ao tratamento em adultos com diabetes tipo 2. A pesquisa contou com 30 pacientes (18 mulheres e 12 homens) entre 33 e 59 anos de idade, matriculados no programa Hiperdia de uma Unidade Básica de Saúde. Os instrumentos utilizados foram os inventários de ansiedade e de depressão de Beck, 


\section{Revisão}

\section{Saúde e Processos Psicossociais}

o inventário de Sintomas de Stress de Lipp (ISSL) e o Questionário geral de saúde e qualidade de vida (SF-36).

Quanto ao estresse, observou-se que não houve diferença significativa entre os sexos, pois $57 \%$ das mulheres e $57 \%$ dos homens obtiverem resultados no ISSL sugerindo presença de estresse.

De um modo geral, o estudo de $\operatorname{Ramos}^{15}$ demonstrou não existir diferença estatisticamente significativa entre os participantes com relação às características sociodemográficas quanto à adesão ao tratamento, nem uma correlação estatisticamente significativa entre esta e a idade e escolaridade destes. Os resultados sugerem que outros fatores, além dos emocionais, devem ser considerados na análise da adesão ao tratamento.

A mensuração do nível de estresse em diabéticos também foi avaliada por Gonçalves ${ }^{16}$. Os dados foram coletados através do Inventário de Sintomas de Stress para Adultos de Lipp, e os principais resultados mostraram que os participantes da pesquisa tinham entre 30 e 82 anos, sendo que a maioria (66\%) realizava tratamento com hipoglicemiantes orais. Apresentaram estresse $79 \%$ dos indivíduos dos quais $50 \%$ encontravam-se na fase de resistência. Os sintomas do estresse mais citados foram os psicológicos (76\%). A predominância do estresse foi maior nas mulheres que nos homens.

Outros pontos pesquisados foram os fatores de risco, o estresse e o cuidado com a saúde realizado pelos trabalhadores de enfermagem diabéticos ${ }^{17}$. O estudo que abordou esta questão foi realizado com 418 profissionais de enfermagem de um hospital universitário. Os dados foram coletados por meio de instrumento semiestruturado e analisado, utilizando-se a distribuição de frequência, teste quiquadrado e odds ratio. A maioria dos profissionais foi do sexo feminino com 20 a 30 anos de idade. Todos os fatores de risco para DM estavam presentes, inclusive os passíveis de modificação.

No estudo desenvolvido por Tavares $^{17}$ houve maior proporção de trabalhadores diabéticos com sobrepeso, hipertensão arterial e glicemia capilar alterada, quando comparados aos que não tinham essa doença. Não se observou maior chance de desenvolver DM entre os trabalhadores de enfermagem que referiram estresse. Os trabalhadores com DM não faziam acompanhamento mensal 


\section{Revisão}

\section{Saúde e Processos Psicossociais}

$(69,2 \%)$, eram atendidos em serviços particulares (70\%) e nem participavam de atividades educativas $(92,3 \%)$.

Um outro estudo, desenvolvido por Silva ${ }^{7}$, avaliou 316 indivíduos com diabetes, sendo $55,4 \%$ do sexo feminino, com idade compreendida entre 16 e 84 anos, sendo 59,8\% com complicações crônicas da doença. Os participantes responderam à Escala de Avaliação de Acontecimentos de vida e à Hospital Anxiety and Depression Scale (HADS) ${ }^{18}$, instrumento de avaliação do estresse através dos acontecimentos de vida ocorridos ao longo do último ano.

Os resultados mostraram que os diabéticos com complicações crônicas não apresentaram maior estresse negativo do que os demais, porém, parecem apresentar menor estresse positivo na sua vida ao longo do último ano.

Já Lessmann ${ }^{4}$ investigou a relação entre estresse e diabetes com 147 mulheres, utilizando a Escala de Estresse Percebido (PSS). Percebeu-se como resultado que houve associação entre as variáveis testadas, sendo possível inferir que as mulheres com estresse elevado são as que vivenciaram sofrimento e apresentam controle glicêmico alterado, ou seja, com valores superiores a 180 $\mathrm{mg} / \mathrm{dL}$. A maioria relatou sofrimento decorrente de problemas nas relações interpessoais, óbito, doença de familiares e solidão.

\section{DISCUSSÃo}

Os achados desta revisão reforçam que há relação entre estresse e diabetes, conforme mostram os resultados de Gonçalves ${ }^{16}$, onde $79 \%$ dos participantes apresentaram estresse, dos quais 50\% encontravam-se na fase de resistência. Os sintomas do estresse mais citados foram os psicológicos $(76 \%)$. A predominância do estresse foi maior nas mulheres que nos homens.

Assim, de acordo com Lessmann ${ }^{4}$, houve associação entre as variáveis testadas, sendo possível inferir que as mulheres com estresse elevado apresentaram controle glicêmico alterado. A maioria relatou sofrimento decorrente de problemas nas relações interpessoais, óbito, doença de familiares e solidão.

Porém, Ramos ${ }^{15}$, em seu estudo, não observou diferença significativa da adesão ao tratamento entre os sexos. Os resultados ainda sugerem que outros 


\section{Revisão}

\section{Saúde e Processos Psicossociais}

fatores, além dos emocionais, devem ser considerados na análise da adesão ao tratamento da diabetes.

Quando comparados aos trabalhadores que não possuem diagnóstico da doença, não se observou maior chance de desenvolver DM entre os trabalhadores de enfermagem que referiram estresse. Houve maior proporção de trabalhadores diabéticos com sobrepeso, hipertensão arterial e glicemia capilar alterada, quando comparados aos que não tinham essa doença. Os trabalhadores com DM não faziam acompanhamento mensal $(69,2 \%)$, eram atendidos em serviços particulares $(70 \%)$ e nem participavam de atividades educativas $(92,3 \%)^{17}$.

Já Silva ${ }^{7}$, analisando os diabéticos com complicações crônicas, percebeu que estes não apresentaram maior estresse negativo do que os demais diabéticos. Porém, parecem apresentar menor estresse positivo na sua vida ao longo do último ano.

Quanto ao tratamento dos pacientes diabéticos que também apresentaram estresse, Cantos $^{9}$ observou que a interação dos pacientes com o grupo, com a equipe multiprofissional de saúde e o acolhimento da equipe mostrou melhoras no estado emocional dos pacientes, fato que talvez possa ser atribuído à mudança no estilo de vida, ao hábito alimentar e ao incentivo à prática de exercício físico, bem como ao abandono de hábitos prejudiciais, como o tabagismo, o sedentarismo, entre outros.

Corroborando com este estudo, Silva ${ }^{7}$ ainda cita que outras pesquisas revelam que pacientes com doenças crônicas como o diabetes têm maior probabilidade de desenvolver formas patológicas de estresse, ansiedade e depressão, podendo apresentar ainda dificuldades no manejo da doença, comprometendo a adesão ao tratamento e consequentemente sua qualidade de vida.

Os estudos apresentados em nosso artigo avaliaram o estresse de maneiras distintas, a metodologia mais empregada nos estudos pesquisados foi o Inventário de Sintomas de Stress para Adultos de Lipp. Além deste, também foram utilizados nos estudos em geral os questionários tolerância ao estresse ${ }^{11}$, vulnerabilidade ao estresse $^{12}$, consciência do estresse ${ }^{13}$, avaliação do estado físico ${ }^{14}$, inventários de ansiedade e de depressão de Beck, questionário geral de saúde e qualidade de 


\section{Revisão}

\section{Saúde e Processos Psicossociais}

vida, Escala de Estresse Percebido e a Escala de Avaliação de Acontecimentos de vida e a Hospital Anxiety and Depression Scale ${ }^{18}$.

\section{CONCLUSÃO}

Em conclusão, o diabetes é uma doença ainda sem tratamento definitivamente satisfatório, não sendo possível evitar integralmente o desenvolvimento de complicações crônicas. É imprescindível que seja desenvolvido um esforço no sentido de melhorar a compreensão do que se pode fazer com que, mesmo em condições difíceis, diabéticos percebam a sua vida como positiva, melhorando também o entendimento sobre a doença e colaborando na prevenção das complicações crônicas.

No que diz respeito ao estresse, há poucos estudos sobre suas consequências e influências em pacientes com DM. Porém, ficou evidente nesta revisão a relação do estresse como fator associado a portadores de DM e a necessidade de medidas que incluam o controle de estresse ao tratamento preconizado para esta doença, com a finalidade de melhorar os níveis glicêmicos e prevenir o aparecimento de complicações.

Cabe ressaltar as dificuldades para a incorporação da mudança de hábitos em pacientes portadores de doenças crônicas em geral. Alguns estudos evidenciam que fatores como a informação, o nível de escolaridade e o acesso a bens e serviços não são suficientes para a motivação e manutenção de mudança de atitudes em relação à saúde. É preciso manter um olhar para a prevenção e minimização de morbidades do DM, sem que estas ações gerem mais conflitos, uma vez que a não adesão aos cuidados e tratamentos pode transcender a vontade de aderir ou não ao que é proposto, estando vinculada também aos seus problemas cotidianos, especialmente ao estresse e ao sofrimento.

\section{REFERÊNCIAS}

1. Brasil. Cadernos de Atenção Básica: Diabetes Mellitus. Brasília, DF: Ministério da Saúde.; 2006.

2. Zagury L, Zagury T, Guidacci J. Diabetes sem medo. Rio de Janeiro: Rocco; 2000. 


\section{Revisão}

\section{Saúde e Processos Psicossociais}

3. Santos JR, Enumo SRF. Adolescentes com Diabetes mellitus tipo 1: seu cotidiano e enfrentamento da doença. Psicol Reflex Crit. 2003;16:411-25.

4. Lessmann JC, Silva DMGV, Nassar SM. Estresse em mulheres com Diabetes mellitus tipo 2. Rev bras enferm. 2011;64:451-6.

5. Leite CC, Halpern A. Síndrome metabólica e diabetes melito. Rev bras hipertens. 2005;12(3):165-8.

6. SBD. Tratamento e Acompanhamento Do Diabetes Mellitus. [S.I.]: Diretrizes SBD. Sociedade Brasileira De Diabetes. ; 2012.

7. Silva I, Pais-Ribeiro J, Cardoso H. Dificuldade em perceber o lado positivo da vida? Stresse em doentes diabéticos com e sem complicações crónicas da doença. Aná Psicológica. 2004;22:597-605.

8. Penteado MS, Oliveira TC. Associação estresse-diabetes mellitus tipo II. Rev Bras Clin Med. 2009;7:40-5.

9. Cantos GA, Souza LC. Acompanhamento de dois pacientes diabéticos por equipe interdisciplinar e multiprofissional. R Eletr de Extensão. 2013;9(14):11.

10. Lipp MEN. Manual do inventário de sintomas de stress para adultos de Lipp (ISSL). São Paulo: Casa do Psicólogo; 2000.

11. Simon RMM. Estresse esclarecendo suas dúvidas. São Paulo: Ahora; 2000.

12. Nahas M. Atividade física, saúde e qualidade de vida: conceitos e sugestões para um estilo de vida ativo. 2 ed. Londrina: Midiograf; 2001.

13. Alves GLB. A consciência do stress. Curitiba-PR: Ed. Souza; 2000.

14. Boucher F, Binette A. Vença o Estresse. São Paulo: Editora Loyola; 1996.

15. Ramos L, Ferreira EAP. Fatores emocionais, qualidade de vida e adesão ao tratamento em adultos com diabetes tipo 2. Rev bras crescimento desenvolv hum $2011 ; 21: 867-77$.

16. Gonçalves AM, Ribas ACLW, Araújo ACC, Silva MS, Caldeira RG, Guedes AC. Mensuração do nível de estresse em indivíduos portadores de diabetes mellitus. J Health Sci Inst. 2008;26(2):173-6.

17. Tavares DMS, Reis NA, Dias FA, Lopes FAM. Diabetes mellitus: fatores de risco, ocorrência e cuidados entre trabalhadores de enfermagem. Acta paul enferm. 2010;23(5):671-6.

18. Zigmond AS, Snaith RP. The hospital anxiety and depression scale. Acta psychiatrica Scandinavica. 1983;67(6):361-70. 